\title{
Different clinical courses with the same findings: two cases of paroxysmal nocturnal hemoglobinuria presenting with thrombocytopenia
}

\author{
Volkan Karakuş, ${ }^{1,2}$ Egemen Kaya, ${ }^{3}$ Yelda Dere, ${ }^{4}$ Fahri Şahinn ${ }^{2,5}$ \\ ${ }^{1}$ Department of Hematology, Alanya Alaaddin Keykubat University, School of Medicine; ${ }^{2}$ PNH Education and Study Group \\ (PESG); ${ }^{3}$ Department of Physiology Muğla Sitkı Koçman University, School of Medicine; ${ }^{4}$ Department of Pathology, Muğla \\ S1tk1 Koçman University Educational and Research Hospital; ${ }^{5}$ Department of Hematology, Ege University, Antalya, Turkey
}

\begin{abstract}
Paroxysmal nocturnal hemoglobinuria (PNH) is a clonal stem cell disease that manifests with chronic intravascular hemolysis, thrombosis, and bone marrow failure. Various degrees of cytopenias accompany the disease. Although laboratory and clinical findings are similar, the disease may show different courses and require different treatments. Herein, we report two different courses of PNH with similar clinical and laboratory findings.
\end{abstract}

\section{Introduction}

Paroxysmal nocturnal hemoglobinuria (PNH) is a clonal hematopoietic stem cell disease characterized by chronic intravascular hemolysis, bone marrow failure, and thrombosis. The disease manifests itself in two main clinical features: hemolytic and hypoplastic. Chronic intravascular hemolytic findings of hemolytic type and pancytopenia in hypoplastic type are the most prominent features. Coombs negative hemolytic anemia (hemolysis laboratory findings), as well as thrombosis, iron deficiency, and/or cytopenic presence, require PNH examination. PNH may be present alone (classical PNH), accompanied by aplastic anemia (AA) and myelodysplastic syndrome. Flow cytometric

Correspondence: Volkan Karakuş, Department of Hematology, Alanya Alaaddin Keykubat University, School of Medicine, 07425, Antalya, Turkey.

Tel.: +90.505.7656778. E-mail: dr_v_karakus@yahoo.com

Key words: Paroxysmal nocturnal hemoglobinuria; thrombocytopenia; aplastic anemia.

Conflict of interests: the authors declare no potential conflict of interests.

Received for publication: 30 May 2020.

Revision received: 11 May 2021.

Accepted for publication: 8 June 2021.

This work is licensed under a Creative Commons Attribution NonCommercial 4.0 License (CC BY-NC 4.0).

${ }^{\circ}$ Copyright: the Author(s), 2021

Licensee PAGEPress, Italy

Italian Journal of Medicine 2021; 15:188-190

doi:10.4081/itjm.2021.1319 analysis of proteins such as CD55, CD59 (linked to the cell membrane by GPI anchor) is the gold standard for the diagnosis of PNH; it is recommended to perform flow cytometric study using at least two surface antibodies in two different cell groups. One of the cell groups must be granulocytes. Clinically apparent hemolysis is unlikely to occur in patients with PNH clonality $<10 \%{ }^{1,2}$

In this article, we, as Paroxysmal Nocturnal Hemoglobinuria Education and Study Group (PESG), report herein two cases presenting with thrombocytopenia and $\mathrm{PNH}$ clone. Especially the second case we present is an ultra-rare condition because of the formation of $\mathrm{PNH}$ clone after Parvovirus B19.

\section{Case Reports}

\section{Case 1}

A 17-year-old male patient with abdominal pain, nausea, and vomiting symptoms for 3-4 days had no past medical or family history. There were no findings on physical examination. Laboratory data showed the following: hemoglobin was $11.8 \mathrm{~g} / \mathrm{dL}$, hematocrit was $37.5 \%$, mean corpuscular volume (MCV) was $105 \mathrm{fL}$, leukocyte count was $2100 / \mathrm{mm}^{3}$, neutrophil count was $1600 / \mathrm{mm}^{3}$, platelet count was $17,000 / \mathrm{mm}^{3}$, lactate dehydrogenase was $367 \mathrm{U} / \mathrm{L}$, urea was $41 \mathrm{mg} / \mathrm{dL}$, creatinine was $0.7 \mathrm{mg} / \mathrm{dL}$, total/indirect bilirubin was $1.88 / 0.57 \mathrm{mg} / \mathrm{dL}$, corrected reticulocyte index was 3.9, and direct/indirect Coombs was (-)/(-). Peripheral smear showed $50 \%$ neutrophils, $42 \%$ lymphocytes, $5 \%$ monocytes, $3 \%$ eosinophils, $1-2$ platelets, and less than one schistocyte in each area. Ebstein Barr virus, cytomegalovirus, Parvovirus B19, hepatitis, and other viral serologies were negative. Ultrasonography and 
abdominal tomography were normal. In the FLAER analysis of the patient with Coombs negative hemolytic anemia, PNH clones were detected: in erythrocytes, type 2: $0.29 \%$ and type 3: $4.39 \%$; in granulocytes: $23.5 \%$, and in monocytes: $16.4 \%$. In the bone marrow examination, which was performed due to the presence of thrombocytopenia without signs of thrombosis, the cellularity was normal, there was erythroid hyperplasia, but dysplastic changes and blast increase were not observed. Just in rare areas, a decrease in cellularity was detected. Cytogenetic analysis showed no anomaly in 46, XY [20]. Eculizumab therapy was started in the patient with PNH diagnosis. After the treatment, nausea and vomiting receded, at the end of the first week, the abdominal pain completely disappeared. In the second year of the treatment, hemoglobin was $11.4 \mathrm{~g} / \mathrm{dL}$, hematocrit was $31.3 \%$, MCV was $96.6 \mathrm{fL}$, leukocyte count was $3820 / \mathrm{mm}^{3}$, neutrophil count was $1560 / \mathrm{mm}^{3}$, platelet count was $39,000 / \mathrm{mm}^{3}$, lactate dehydrogenase (LDH) was $286 \mathrm{U} / \mathrm{L}$. The patient did not need any transfusion, did not have bleeding, thrombosis complication, hemolytic attack, and follow up has been continued with medication.

\section{Case 2}

A 22-year-old woman had complaints of vaginal bleeding and palpitation for 3-4 days. In the physical examination of the patient, there was a petechial rash in the lower extremities. Laboratory data showed the following: hemoglobin was $7.9 \mathrm{~g} / \mathrm{dL}$, hematocrit was $23.1 \%$, MCV was $93.5 \mathrm{fl}$, leukocyte count was $3980 / \mathrm{mm}^{3}$, neutrophil count was $2980 / \mathrm{mm}^{3}$, platelet count was $8000 / \mathrm{mm}^{3}$, LDH was $263 \mathrm{U} / \mathrm{L}$, urea was 29 $\mathrm{mg} / \mathrm{dl}$, creatinine was $0.51 \mathrm{mg} / \mathrm{dL}$, total/indirect bilirubins were $0.43 / 0.2 \mathrm{mg} / \mathrm{dL}$, corrected reticulocyte index was 2.9, direct/indirect Coombs was (-)/(-). Peripheral smear showed $76 \%$ neutrophil, $20 \%$ lymphocyte, $2 \%$ monocyte, $2 \%$ eosinophil, $1-2$ platelets, and less than one schistocyte in each area. Parvovirus B19 IgM was (-), IgG was (+); Ebstein Barr Virus, cytomegalovirus, hepatitis, and other viral serologies were negative. Abdominal ultrasonography was normal. In the FLAER analysis of the patient with Coombs negative hemolytic anemia, $\mathrm{PNH}$ clones were detected: in erythrocytes, type 2: $0.02 \%$, and type 3 $0.27 \%$, in granulocytes: $3.06 \%$, in monocytes: $5.06 \%$. In the bone marrow biopsy, cellularity was normal $(70 \%)$, advanced hypoplasia in megakaryocytes and erythroid hyperplasia were detected. In the cytogenetic analysis in 46, XX [10], nonclonal numerical anomaly, diploidy [5], and hypodiploidy [1] were found. Eculizumab therapy was started for the patient having a diagnosis of PNH. Bone marrow biopsy was repeated 6 weeks later due to the absence of clinical response, continuing anemia, and thrombocytopenia.
Upon $10 \%$ cellularity and megakaryocytes were not seen, treatment with ATG and cyclosporine was also added to the patient who needed apheresis, platelet, and erythrocyte transfusion. Unrelated allogeneic hematopoietic cell transplantation was planned for the patient, who could not receive a response to the treatment and did not have a suitable donor.

\section{Discussion}

The incidence of AA is $70 \%$ in children and adolescents with PNH disease, and PNH clones are observed in $15-25 \%$ of aplastic anemias. ${ }^{3,4}$ Acquired AA is a prototype of bone marrow insufficiency caused by immune-mediated destruction of hematopoietic stem and/or precursor cells, in which the autoreactive $\mathrm{T}$ cells are expected to play a central role. However, it has been understood that AA is more complex than a simple immune-mediated bone marrow failure, and the reason is that this disease is late clonal. Clonal hematopoiesis is detected in more than $50 \%$ of AA patients. The high incidence of MDS, AML, and PNH among AA patients prior to the immunosuppressive therapy is remarkable.

Frequent development of diseases such as paroxysmal nocturnal hemoglobinuria and myelodysplastic syndromes/acute myeloid leukemia suggest that there is a late clonal disease. ${ }^{4}$ In their study, Yoshizato et al. revealed that the burden of alleles of mutations changed and developed over the years. They have shown that in most cases, small clones retain their size for years. However, MDS/AML transformation has been shown to be associated with an increased size of the dominant clone and the addition of new clones. ${ }^{5}$

Kulagin et al. demonstrated that the presence of PNH clones accompanying patients with aplastic anemia predicts the response to immunosuppressive therapy. In the presence of the PNH clone, response rates to immune suppressive therapy were better, and even reticulocytosis was an independent predictor of good response. ${ }^{6}$

There are three clinical manifestations of paroxysmal nocturnal hemoglobinuria: classical, subclinical, and aplastic, and the form associated with aplastic anemia is usually associated with less than $10 \% \mathrm{PNH}$ clones. It is usually accompanied by other cytopenias and may include hemolysis findings. In general, bone marrow failure is predominant. Treatment of aplastic anemia-associated PNH is primarily based on the treatment of underlying bone marrow failure syndrome, which requires immunosuppressive therapy or an allogeneic bone marrow transplantation. ${ }^{7}$ In allogeneic stem cell transplantation, the results of lowdensity transplants have been shown to be effective in destroying the PNH clone. ${ }^{8}$ There are publications reporting that adding eculizumab to immune suppres- 
sive therapy in the PNH subtype associated with aplastic anemia is effective without a hemolytic attack. ${ }^{9}$

\section{Conclusions}

We describe herein two cases, a classic one presented with thrombocytopenia initially and an aplastic anemia-related PNH. In the second case, the lack of eculizumab response led to early bone marrow control, suggesting that rapid aplastic anemia might have developed, although the first bone marrow cellularity was normal. This suggests that early bone marrow control should be performed, and treatment should be revised accordingly, especially if PNH cases with low clones and multiple cytopenias fail to respond, independently of the initial bone marrow cellularity.

\section{References}

1. Sahin F, Akay OM, Ayer M, et al. Pesg PNH diagnosis, follow-up and treatment guidelines. Am J Blood Res 2016;6:19-27.

2. Borowitz MJ, Craig FE, Digiuseppe JA, et al. Guidelines for the diagnosis and monitoring of paroxysmal nocturnal hemoglobinuria and related disorders by flow cytometry. Cytometry B Clin Cytom 2010;78:211-30.

3. Kulagin A, Lisukov I, Ivanova M, et al. Prognostic value of paroxysmal nocturnal haemoglobinuria clone presence in aplasticanaemia patients treated with combined immunosuppression: results of two-centreprospective study. Br J Haematol 2014;164:546-54.

4. Ogawa S. Clonal hematopoiesis in acquired aplastic anemia. Blood. 2016;128:337-47.

5. Yoshizato T, Dumitriu B, Hosokawa K, et al. Somatic mutations and clonal hematopoiesis in aplastic anemia. N Engl J Med 2015;373:35-47.

6. Kulagin A, Lisukov I, Ivanova M, et al. Prognostic value of paroxysmal nocturnal haemoglobinuria clone presence in aplastic anaemia patients treated with combined immunosuppression: results of two-centre prospective study. Br J Haematol 2014;164:546-54.

7. Devalet B, Mullier F, Chatelain B, et al. Pathophysiology, diagnosis, and treatment of paroxysmal nocturnal hemoglobinuria: a review. Eur J Haematol 2015;95:190-8.

8. Lee SE, Park SS, Jeon YW, et al. Outcomes of allogeneic stem cell transplantation in patients with paroxysmal nocturnal hemoglobinuria with or without aplastic anemia. Eur J Haematol 2017;99:336-43.

9. Asano J, Ueda R, Tanaka Y, et al. Effects of immunosuppressive therapy in a patient with aplastic anemiaparoxysmal nocturnal hemoglobinuria (AA-PNH) syndrome during ongoing eculizumab treatment. Intern Med 2014;53:125-8. 